\title{
Rapid quantification of myocardial fibrosis: a new macro-based automated analysis
}

\author{
Awal M. Hadi • Koen T. B. Mouchaers • Ingrid Schalij • Katrien Grunberg • \\ Gerrit A. Meijer • Anton Vonk-Noordegraaf • Willem J. van der Laarse • \\ Jeroen A. M. Beliën
}

Accepted: 30 September 2010 /Published online: 3 May 2011

(C) The Author(s) 2011. This article is published with open access at Springerlink.com

\begin{abstract}
Background Fibrosis is associated with various cardiac pathologies and dysfunction. Current quantification methods are time-consuming and laborious. We describe a semiautomated quantification technique for myocardial fibrosis and validated this using traditional methods.

Methods Pulmonary Hypertension (PH) was induced in adult Wistar rats by subcutaneous monocrotaline (MCT)

This paper is a reprint from rapid quantification of myocardial fibrosis: a new macro-based automated analysis, Awal M. Hadi, Koen T.B. Mouchaers, Ingrid Schalij, Katrien Grunberg, Gerrit A. Meijer, Anton Vonk-Noordegraaf, Willem J. van der Laarse and Jeroen A.M. Belien, originally published in Analytical Cellular Pathology/Cellular Oncology, Volume 33, number 5-6, 2010, pp. 257-269, IOS Press.
\end{abstract}

Electronic supplementary material The online version of this article (doi:10.1007/s13402-011-0035-7) contains supplementary material, which is available to authorized users.

\footnotetext{
A. M. Hadi • K. T. B. Mouchaers · I. Schalij •

A. Vonk-Noordegraaf

Department of Pulmonary Diseases,

Institute for Cardiovascular Research,

VU University Medical Center,

Room 0 E 50, P.O. Box 7057,

1007 MB Amsterdam,

The Netherlands
}

K. Grunberg • G. A. Meijer • J. A. M. Beliën $(\bowtie)$

Department of Pathology, Institute for Cardiovascular Research,

VU University Medical Center,

Room 0 E 50, P.O. Box 7057, 1007 MB Amsterdam,

The Netherlands

e-mail: JAM.Belien@vumc.nl

W. J. van der Laarse

Department of Physiology, Institute for Cardiovascular Research,

VU University Medical Center,

Room 0 E 50, P.O. Box 7057, 1007 MB Amsterdam,

The Netherlands injection(40 $\mathrm{mg} / \mathrm{kg})$. Cryosections of myocardial tissue $(5 \mu \mathrm{m})$ of $\mathrm{PH}$ rats $(n=9)$ and controls $(n=9)$ were stained using Picrosirius red and scanned with a digital microscopic MIRAX slide scanner. From these sections 21 images were taken randomly of each heart. Using ImageJ software a macro for automated image analysis of the amount of fibrosis was developed. For comparison, fibrosis was quantified using traditional polarisation microscopy. Both methods were correlated and validated against stereology as the gold standard. Furthermore, the method was tested in paraffin-embedded human tissues.

Results Automated analysis showed a significant increase of fibrosis in PH hearts vs. control. Automated analysis correlated with traditional polarisation and stereology analysis $\left(r^{2}=0.92\right.$ and $r^{2}=0.95$ respectively). In human heart, lungs, kidney, and liver, a similar correlation with stereology $\left(r^{2}=0.91\right)$ was observed. Time required for automated analysis was $22 \%$ and $33 \%$ of the time needed for stereology and polarisation analysis respectively.

Conclusion Automated quantification of fibrosis is feasible, objective, and time-efficient.

Keywords Collagen · Fibrosis · Slide scanner - Whole slide image $\cdot$ Image analysis · Open source $\cdot$ Picrosirius red

Polarised light $\cdot$ Stereology

\section{Introduction}

Collagen fibers play an important role in sustaining structural integrity of healthy tissues. More crucially they play a vital role in pathological processes like inflammation and after tissue injury. For instance, chronic inflammation, irrespective of cause and organ affected, often results in fibrosis, i.e. an excess of connective tissue [46]. This 
process is of particular importance in the heart as it is involved in numerous different cardiac diseases[39]. In the diabetic or hypertensive heart disease for example, the myocardium of the left ventricle (LV) undergoes drastic structural remodeling. Activation of neurohumoral pathways, like TGF- $\beta$, endothelin-1, or Angiotensin II, stimulate the transition of fibroblasts into myofibroblasts and enhance the production of extracellular matrix (ECM) components in both ventricles in these hearts [39, 48]. This not only affects myocardial structure, but also leads to stiffening of the ventricle and altered signaling to cardiomyocytes, known to contribute to both systolic and diastolic heart failure [2, 39-41, 43]. Similarly, in pulmonary hypertension $(\mathrm{PH})$, the right ventricle (RV) of the heart is subjected to pressure overload causing activation of fibroblasts and subsequent RV dysfunction $[15,24]$. Cardiac arrhythmias, like atrial fibrillation, are known to originate from a disturbed intercellular signaling due to fibrosis [5, 12]. In many cardiac diseases, histological changes of the myocardium, including fibrosis, have been linked to cardiac function and even survival $[29,40]$.

One of the most widely used methods to visualize fibrosis in histological tissue is by staining it with Picrosirius red [21, 25, 32, 37, 45]. Picrosirius red, in contrast to more traditional stains like van Gieson and trichrome, has selectivity which makes it ideal and superior for both staining and quantification of collagen [44, 45]. Moreover, known issues by traditional methods like staining variability and the tendency of stain to fade is absent when using Picrosirius red. Picrosirius red enhances the birefringence properties of collagen which makes the collagen fibers easy to detect using circularly polarised light [13, 32, 45]. Quantification of fibrosis subsequently can be performed with stereology. This conventional manual planimetry method has proven its value but it is laborious, and the result depends in part on the skill of the observer.

Semi-quantitative and semi-automated methods of fibrosis quantification have been introduced [3, 28, 45, 47]. However, detailed literature on methodological and technical aspects of fibrosis quantification is scarce. For a more precise analysis of the associations between extent of fibrosis on the one hand, and pathophysiology and clinical phenotype on the other, there is a clear need for better tools for quantifying fibrosis in tissue sections. To achieve this we investigated whether it would be feasible to quantify interstitial myocardial fibrosis using a semi-automated image analysis technique that would be reproducible, less sensitive to user interaction and reduces time needed for analysis. To this end, we employed the use of high-speed, automated whole slide imaging (WSI) system (also called digital slide system or virtual microscope) to convert ordinary glass slides into digital slides. Subsequent automated analysis of the captured images was carried out using a ImageJ macrobased algorithm that identifies, separates and quantifies Picrosirius red stain representing fibrosis.

\section{Materials and methods}

\subsection{Experimental model}

We used the rat model monocrotaline (MCT) induced $\mathrm{PH}$, which is known to induce the production of myocardial fibrosis due to RV pressure overload [7, 18, $24,36]$. PH was induced in male Wistar rats $(n=9)$ by a single injection of MCT $(40 \mathrm{mg} / \mathrm{kg}$ body mass s.c., SigmaAldrich, Zwijndrecht, The Netherlands) at approximately $180 \mathrm{~g}$ body mass. MCT was dissolved in sterile saline $(8 \mathrm{mg} / \mathrm{ml})$ at $\mathrm{pH}$ 7.4. Control rats $(n=9)$ received an equal volume of saline. After initial single MCT injection $\mathrm{PH}$ development was maintained for 25 days with no further interventions. All experiments were approved by the Institutional Animal Care and Use Committee and conform the Helsinki convention for the use and care of animals.

\subsection{Echocardiographic measurements}

Echocardiographic measurements were performed to evaluate and to confirm clinical signs of MCT induced $\mathrm{PH}$. At day 0 and 25 trans-thoracic echocardiography (TTE) was performed on anaesthetized but spontaneously breathing rats ( $4 \%$ isoflurane in air maintained at $1.5 \%)$ using an Aloka SSD4000 ultrasonographic system equipped with a $13.5 \mathrm{MHz}$ transducer (Biomedic, Almere, The Netherlands). A single-lead electrocardiogram was obtained by placing electrodes on the upper and lower limbs. Heart rate (HR) was recorded for at least 3 cardiac cycles. The morphologies of RV and LV was assessed and measurements of end-diastolic diameter (EDD) and end-systolic diameter (ESD) were made as described before by Hardziyenka et al. [17]and Salemi et al. [35] respectively. All measurement were done according to the recommendations and standards of the American Society of Echocardiography [33].

RV and LV end-systolic and end-diastolic diameters (ESD and EDD respectively) of 3 representative cardiac cycles in 4 different recordings were stored (Scion Image version 4.0.3.2, Scioncorp, Maryland, USA). Average values were used to calculate RV and LV fractional shortening $(\mathrm{FS}): \mathrm{FS}=((\mathrm{EDD}-\mathrm{ESD}) / \mathrm{EDD}) \times 100 \%$. In addition average values of the LVEDD and LVESD were used to calculate stroke volume (SV) and cardiac output 
(CO) by calculating end-systolic and end-diastolic volumes assuming the LV as a prolate spheroid [10, 31].

\subsection{Hemodynamic measurements}

Before euthanisation, the RV systolic pressure (RVSP) was measured using a Millar pressure catheter (Millar) by direct insertion through the RV walls after thoracotomy. Data were obtained using a Power Lab setup (AD Instruments, New South Wales, Australia). Systemic blood pressure was monitored using a fluid-filled catheter inserted into the left carotid artery. Body temperature was monitored and maintained at $37^{\circ} \mathrm{C}$ with a heating pad.

Pulmonary vascular resistance (PVR) was estimated according to Poiseuille's law: PVR $\approx[$ mean PAP $] /[$ cardiac output $] \approx$ $(0.61 \times$ [systolic $\mathrm{RV}$ pressure] $+2 \mathrm{mmHg}) /$ [cardiac output] $[6,16,27]$.

\subsection{Histochemistry}

\subsubsection{Animal tissue}

After isoflurane anesthesia, animals were euthanized by excision of the hearts. Subsequently hearts were perfused with Tyrode solution to remove blood [9]. Hearts were frozen in liquid nitrogen, and cryosections $(5 \mu \mathrm{m})$ were air-dried for $20 \mathrm{~min}$. Direct staining of cryosections with Picrosirius red caused a strong background staining of cardiomyocytes. To remove background staining we treated the sections as if they were paraffin embedded sections. Air-dried sections were immersed in xylene for $10 \mathrm{~min}$ at room temperature and transferred to water through descending concentrations of ethanol $(100 \%, 96 \%, 80 \%$ and $70 \%$, all 10 s). Staining was performed using $0.1 \%$ solution of Sirius red F3BA in saturated aqueous solution of picric acid for one hour at $25^{\circ} \mathrm{C}$ [21, 32]. Subsequently, sections were rinsed in $0.01 \mathrm{~N} \mathrm{HCl}$ for $2 \mathrm{~min}$. Sections were dehydrated in ascending concentrations of ethanol $(70 \%, 80 \%, 96 \%$ and $100 \%$, each $10 \mathrm{sec}$ ) and cleared in two stages in xylene, $10 \mathrm{~min}$ each. Sections were covered with Entellan mounting medium (Merck, Darmstadt, Germany) and a glass cover slip.

\subsubsection{Human tissue}

Post-mortem tissue samples of a 78 year old male patient with known $\mathrm{PH}$ in his medical history were obtained. From the paraffin embedded tissue samples of this patient, myocardial tissue, lungs, kidney and liver sections were cut and stained with Picrosirius red as described above.
2.5 Digital slide scanner, image acquisition, processing and analysis

\subsubsection{Mirax scan}

Picrosirius red stained sections were automatically scanned with a digital (microscopic) Mirax slide Scanner system (3DHISTECH, Budapest, Hungary) equipped with a $20 \mathrm{x}$ objective with a numerical aperture of 0.75 and a Sony DFW-X710 Fire Wire 1/3" type progressive SCAN IT CCD (pixel size $4.65 \times 4.65 \mu \mathrm{m}$ ). The actual scan resolution (effective pixel size in the sample plane) at $20 \times$ is $0.23 \mu \mathrm{m}$. The accompanying software allows the user to navigate through the captured Whole Slide Image (WSI) at any given digital zoom up to 540X. The experimenter can mark areas to be excluded, like staining artefacts or e.g. perivascular areas, by placing an annotation in the image acquired for analysis. Bright green colour was used to facilitate automatic detection of an annotation by the image processing macro in ImageJ (Fig. 1).

\subsubsection{Quantification of fibrosis using semi-automated image analysis}

After scanning the Picrosirius red sections with the Mirax scanner, 21 images of a section at $20 \mathrm{X}$ magnification (images taken at full resolution with a single image dimension set at $1,024 \times 768$ pixels, pixel size is $0.23 \mu \mathrm{m} \times 0.23 \mu \mathrm{m}$ ) were obtained randomly from the $\mathrm{LV}$, the RV, and the septum from across the entire digital slide as described above. The Images were subsequently analysed using the batch mode of the ImageJ macro.

\subsubsection{Macro for ImageJ image analysis}

All images were analysed using an in-house developed macro, which requires ImageJ v1.38 or higher. This ImageJ macro was written to quantify the percentage of fibrosis compared to the total amount of tissue within an image. This percentage was assessed by determining the total tissue areas occupied by cardiomyocytes and collagen and excluding the lumen (i.e. empty spaces), as well as excluding annotations of perivascular fibrosis and staining artefacts (areas demarcated bright green by the user, in order to be recognized by the macro). The colour thresholding algorithm used by this macro is based on an algorithm written by G. Landini (version v1.8) available at: [http://www.dentistry.bham.ac.uk/landinig/software/software. html].

For all images to be analysed, the macro first applies a colour transformation from the RGB colour space to the CIE LAB colour space. Then for each specific tissue compartment (for instance lumen, cardiomyocytes or collagen fibers) the macro applies specific thresholds. All 


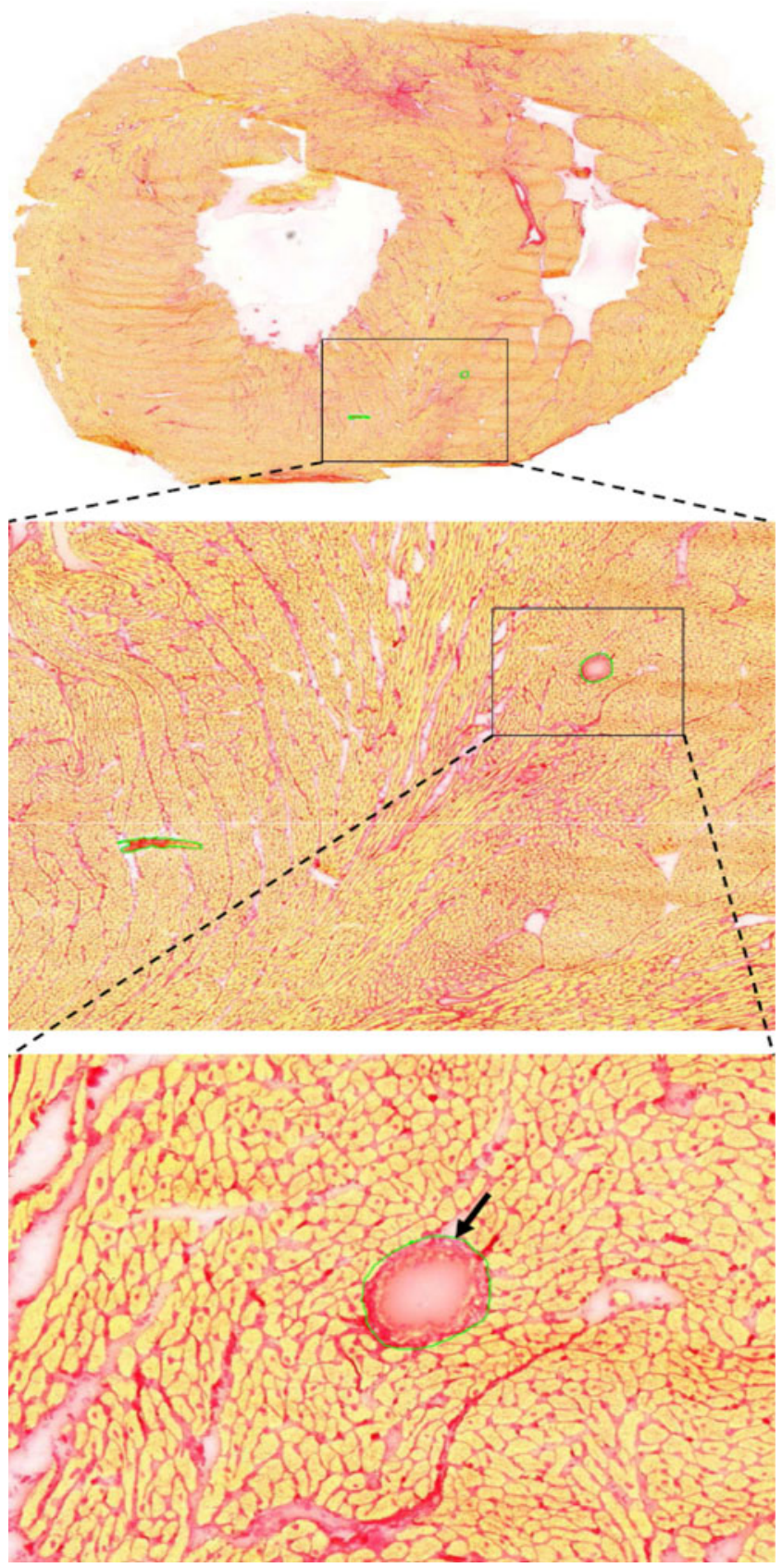

Fig. 1 The Mirax scan was used to scan entire sections of Picrosirius red stained myocardial tissue. The Mirax Viewer software allows the user to navigate through the section and zoom until the desired magnification used for analyses of fibrosis. If the user wishes to exclude areas, like staining artifacts or vessels, the user can apply annotations to the image by demarcating them in (in our study) green (arrow)

thresholds used by the macro were set empirically by analysing a test set of 63 images (RV, LV and Septum) and selecting the threshold which identifies the components best. This was independently done by AMH, KTBM and JAMB. These empirically determined thresholds were recorded in the macro to be applied on all images to be analyzed. Once the macro is loaded onto the Image-J software and executed, the experimenter is requested to select the image to be analysed (Fig. 2a). First the macro detects the annotated areas to be excluded from the analysis (Figs. 1 and 2b). In this case the macro detects the green demarcation line by specifically thresholding the demarcation colour. Next, every pixel inside the demarcated area will be assigned to the area to be excluded from the analysis. Then the algorithm determines the "total tissue component" (i.e. all the information in the image which does not represent the annotated areas (Fig. 2b) or lumen (Fig. 2c)). In short, the macro first determines the lumen part (i.e. the empty spaces in de the image where there is no staining at all). The lumen masked image is then inverted and from this image the area to be excluded is subtracted, resulting in the 'total tissue component'. The total tissue component is split in a part containing fibrosis and a remaining part (i.e. cardiomyocytes and other tissue components than fibrosis). The algorithm detects the fibrosis by setting thresholds specific for the Picrosirius red stained component. The detected fibrosis components in the image are presented in a temporary "fibrosis" image (Fig. 2d). The algorithm then assesses the remaining cardiomyocytes, by classifying the remaining area, by subtracting the exclusion areas, lumen and fibrosis from the entire image, and presents the results in a temporary "cardiomyocyte" image (Fig. 2e). After obtaining all the individual components and correcting the images for the areas to be excluded, the macro assesses the areas of all components in pixels, presents them in a table to the user who can then assess the percentage of fibrosis (i.e. area of fibrosis/(area of cardiomyocytes + area of fibrosis) $\mathrm{x} 100$ ) for the image analysed (Fig. 2f).

The macro is able to analyse multiple images in a row (batch mode). This batch version opens each image in a folder, processes them as described above and writes the results for each image on a separate line to a semi-colon separated output file. The macro as outlined above (also the batch mode) is available as online supplement.

\subsubsection{Quantification of fibrosis using polarised light microscopy image analysis}

The same sections in the same 21 areas described above were studied (using the original slides) with a Leica DMRB microscope (Wetzlar, Germany) fitted with polarisation filters. Circularly polarised light was obtained as described by Frohlich [13] and Whittaker [45]. Images were obtained with a $20 \mathrm{X}$ objective and a monochrome charge-coupled device camera (Sony XC-77CE; Towada, Japan) connected to an LG-3 frame grabber (Scion; Frederick, MD) and analysed using ImageJ v. 1.41a, developed for microscopy (USA National Institute of Health, http://www.macbiophotonics. 
Fig. 2 This figure shows the different steps of the ImageJ macro in identifying different structures in a (a) Picrosiriusred stained myocardial crossection. (b) First the macro determines the area to excluded which is demarcated by the investigator. (c) Subsequently the areas not occupied by structures are analysed and determined as lumen. (d) The macro then analyses the area occupied by fibrosis and by subtracting these values, the area occupied by (e) cardiomyocytes (in this study; this image contains all tissue compartments left) is determined. At last (f) the results in pixels are presented to the user

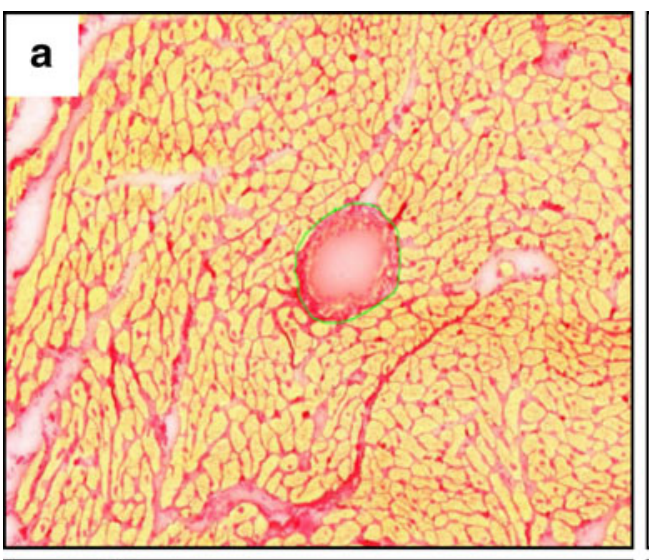

b
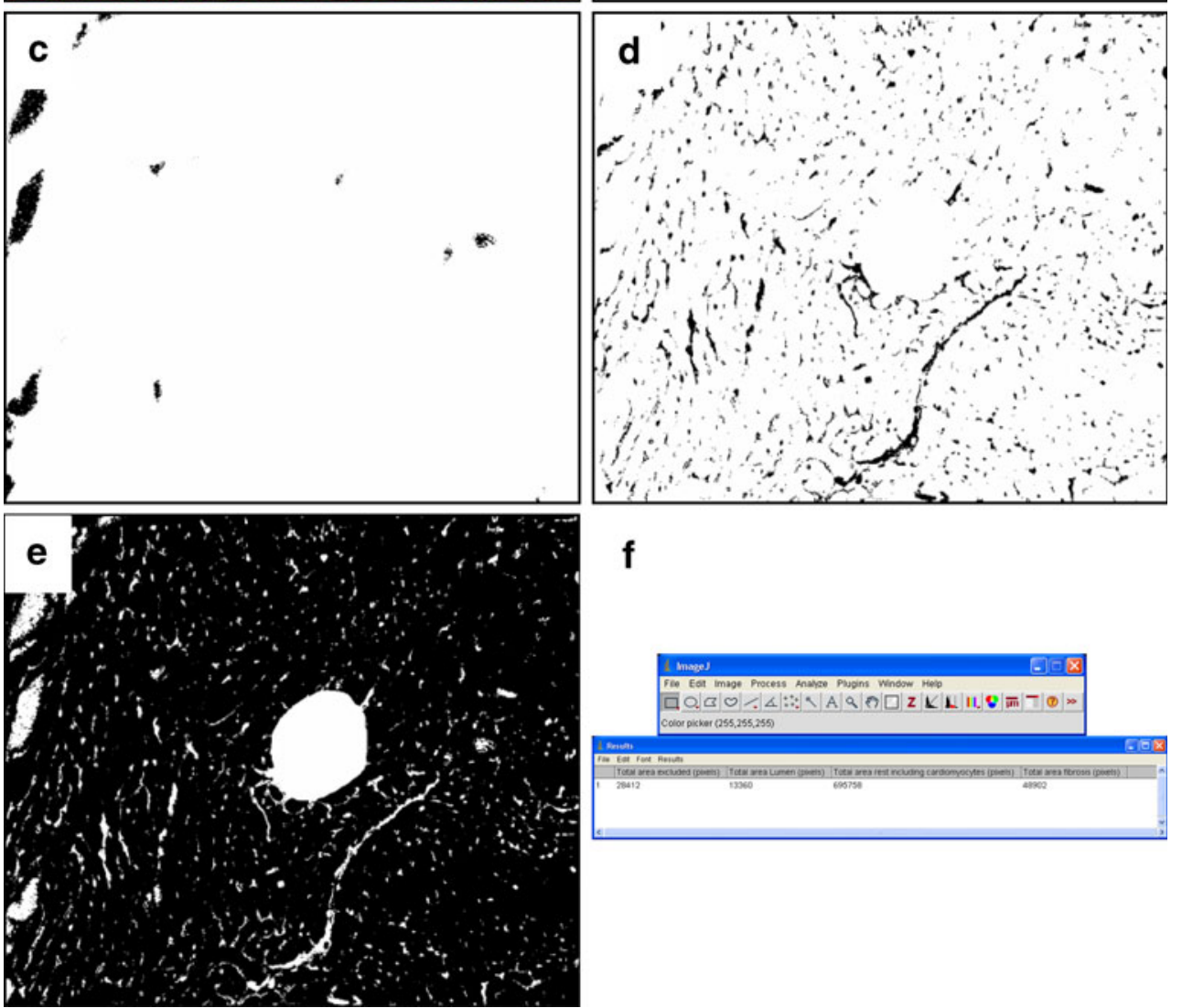

f

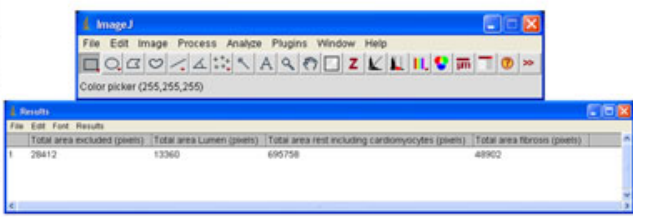

ca/imagej/). The effective pixel size at 20x is $0.25 \times 0.25 \mu \mathrm{m}$ and the image size in pixels $756(\mathrm{H}) \times 581(\mathrm{~V})$. A threshold was set only showing areas occupied by collagen, and subsequently binary images were made. The relative area occupied by collagen is then calculated in proportion to the total area (Fig. 3a, b, c).

\subsubsection{Quantification of fibrosis using stereology}

Stereology served as gold standard to validate the methods used. To that end we used the Q-prodit software (Leica) to perform stereology. This software generates a $0.29 \mathrm{~mm}$ by $0.72 \mathrm{~mm}$ (area $0.2088 \mathrm{~mm}^{2}$ at the section level) 200-points Weibel - grid for a 20x objective with numerical aperture of
0.5. The grid was superimposed on images of 7 randomly selected areas of 5 sections (in a total of 35 areas), a subset of the same sections as well as areas of each section which were previously analysed by the two methods described earlier. The number of intersections between collagen fibres and the grid within the reference area were noted and the relative area occupied by collagen was calculated.

\subsection{Statistics}

All values of hemodynamic and echocardiographic parameters were averaged, and mean $\pm \mathrm{SE}$ given. From a total of 18 RVSP measurements, $89 \%$ was successful (control $n=7$, 

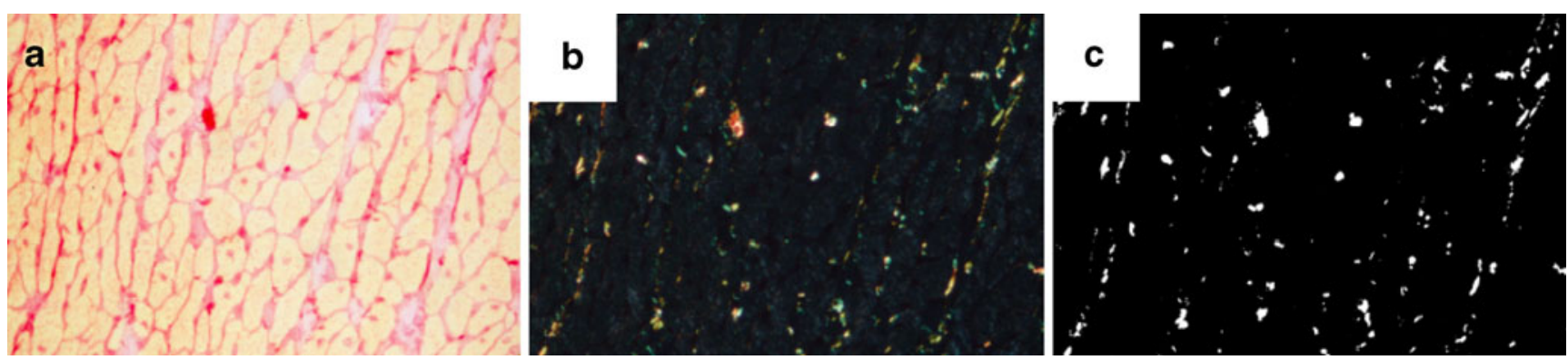

Fig. 3 This figure shows images used in the quantification of fibrosis using polarisation light microscopy. (a) Shows a typical example of a Picrosirius red stained section of myocardium. (b) Using polarisation

MCT $n=9$ ). RV echocardiography was successfully performed in all rats. 94\% of LV echocardiography was successfully performed (control $n=8$, MCT $n=9$ ).

All other data, unless otherwise stated, are presented as mean \pm SEM. Student's $t$-test was used to define differences between groups. Pearson's correlation analysis was used to detect correlations between the different methods. $p<0.05$ was considered to be statistically significant.

\section{Results}

3.1 Hemodynamic and echocardiographic parameters of MCT induced $\mathrm{PH}$

Table 1 shows hemodynamic and echocardiographic parameters as well as body and lung masses of the filters, only polarised light is passed through which can be transferred into (c) a binary image, used for calculation of the percentage fibrosis per area

groups of animals studied. MCT clearly induced PAH, which was signified by the development of the first clinical signs. On day $25 \mathrm{RV}$ systolic pressure has risen to $62 \pm 7 \mathrm{mmHg}$ from $28 \pm 2 \mathrm{mmHg}$ at baseline in control rats $(p<0.05$ vs control). During this period there was a marked drop in cardiac output (CO) from $116 \pm 10 \mathrm{ml} / \mathrm{min}$ at baseline to $71 \pm 10 \mathrm{ml} / \mathrm{min}$ ( $p<0.05$ vs control). In addition PVR increased from $15,120 \pm 540 \mathrm{dyn} \cdot \mathrm{s} \cdot \mathrm{cm}^{-5}$ at baseline, to $46,025 \pm 578 \mathrm{dyn} \cdot \mathrm{s} \cdot \mathrm{cm}^{-5}$ on day $25(p<0.05 \mathrm{vs}$ control. The poor clinical and hemodynamic condition of the MCT induced PAH rats was confirmed by dramatic drop in body mass from $363 \pm 9 \mathrm{~g}$ on day 0 to $311 \pm 12 \mathrm{~g}$ ( $10 \%$ body mass loss) on day 25 ( $\mathrm{p}<0.05$ vs control). A subsequent simultaneous increase in wet lung mass was also observed in MCT treated rats compared to control.

In addition echocardiographic data in Table 1 showed marked dilation of the RV in MCT rats as demonstrated by increased RVEDDs $(4.18 \pm 0.52 \mathrm{~mm}$ vs $2.48 \pm 0.05 \mathrm{~mm}, p<$

Table 1 Hemodynamic and echocardiographic data for the RV and LV of control and MCT treated rats are presented as well as values for body and lung mass. In addition, p-values are given for the variance for these parameters

\begin{tabular}{|c|c|c|c|c|}
\hline & Control & MCT & $\mathrm{p}$ value vs. Control & Summary \\
\hline Body mass (g) & $361 \pm 20$ & $310 \pm 7$ & $\mathrm{p}<0.05$ & $*$ \\
\hline Lung mass (g) & $1.71 \pm 0.21$ & $2.43 \pm 0.10$ & $\mathrm{p}<0.05$ & * \\
\hline Heart rate $(\mathrm{BPM})$ & $391 \pm 75$ & $339 \pm 21$ & $\mathrm{p}<0.05$ & $*$ \\
\hline Cardiac output (ml/min) & $116 \pm 10$ & $71 \pm 10$ & $\mathrm{p}<0.05$ & $*$ \\
\hline $\operatorname{PVR}\left(\right.$ dyn $\left.\cdot \mathrm{s} \cdot \mathrm{cm}^{-5}\right)$ & $15,120 \pm 540$ & $46,025 \pm 578$ & $\mathrm{p}<0.05$ & * \\
\hline RVSP (mmHg) & $29 \pm 1$ & $63 \pm 6$ & $\mathrm{p}<0.05$ & * \\
\hline RVEDD (mm) & $2.66 \pm 0.04$ & $4.20 \pm 0.45$ & $\mathrm{p}<0.05$ & * \\
\hline RVESD (mm) & $1.09 \pm 0.03$ & $2.40 \pm 0.24$ & $\mathrm{p}<0.05$ & * \\
\hline RV FS (\%) & $56 \pm 7$ & $43 \pm 4$ & $\mathrm{p}<0.05$ & * \\
\hline LVEDD (mm) & $6.87 \pm 0.20$ & $5.66 \pm 0.34$ & $\mathrm{p}<0.05$ & $*$ \\
\hline LVESD (mm) & $3.09 \pm 0.17$ & $2.50 \pm 0.20$ & $\mathrm{p}<0.05$ & $*$ \\
\hline LV FS (\%) & $56 \pm 2$ & $61 \pm 2$ & $\mathrm{p}>0.05$ & NS \\
\hline Systemic pressure $(\mathrm{mmHg})$ & $90 \pm 1$ & $71 \pm 3$ & $\mathrm{p}<0.05$ & $*$ \\
\hline
\end{tabular}

n=7-9 rats/group. MCT monocrotaline; RV right ventricle; LV left ventricle; RVSP RV systolic pressure; RVEDD and LVEDD, RV and LV enddiastolic diameter respectively; RVESD and LVESD, RV and LV end-systolic diameter respectively; PVR Pulmonary Vascular Resistance; FS fractional shortening; NS not significant. ${ }^{*} p<0.05$ vs. control rats. Values are means $\pm \mathrm{SE}$ 
0.05 vs control) and RVESDs $(2.39 \pm 0.38 \mathrm{~mm}$ vs $1.07 \pm$ $0.06 \mathrm{~mm}, p<0.05$ vs control). Calculation of RV FS revealed a further significant decrease in $\mathrm{PH}$ induced MCT rats compared to control ( $44 \pm 3 \%$ vs $57 \pm 3 \%, p<0.05$ vs control).

\subsection{Quantification of fibrosis using circularly polarized} light microscopy image analysis

Analyses of Picrosirius red stained sections using polarisation microscopy revealed a significant increase in the amount of fibrosis in the RV of MCT rats $(3.02 \pm 0.20 \%)$ compared to control $(1.36 \pm 0.09 \%, p<0.05)$. This significant increase in fibrosis was also observed in the LV $(2.72 \pm 0.19 \%)$ vs control $(1.21 \pm 0.20 \%, p<0.05)$ and in the septum $(2.50 \pm 0.17 \%)$ vs control $(1.00 \pm 0.07 \%, \mathrm{p}<0.05)$, (Fig. 4 a, b, c).
3.3 Quantification of fibrosis using semi-automated image analyses

Analysis of the same areas using the ImageJ macro revealed a similar pattern, showing significant increase of fibrosis in RV of MCT rats compared to control (4.03 \pm $0.73 \%$ vs $1.58 \pm 0.19 \%, p<0.05)$, in the LV compared to control $(3.20 \pm 0.45 \%$ vs $1.22 \pm 0.10 \%, p<0.05)$ and in de septum compared to control $(2.63 \pm 0.38 \%$ vs $1.04 \pm 0.12 \%$, $p<0.05$ ), (Fig. 4d, e, f).

\subsection{Correlation of measurements with stereology}

7 areas each of the RV, LV and the septum (a total of 21 areas previously examined in the above mentioned methods) were randomly selected in 5 cryosections and analysed
Fig. 4 Polarisation and ImageJ analyses showed that hearts of $\mathrm{PH}$ rats revealed more fibrosis compared to control. Note the increase in collagen content was not limited to the RV only, but was also found in the LV and the septum of these hearts. Note also that Image $J$ analyses detected more fibrosis than the method of polarization

\section{Polarisation}

a

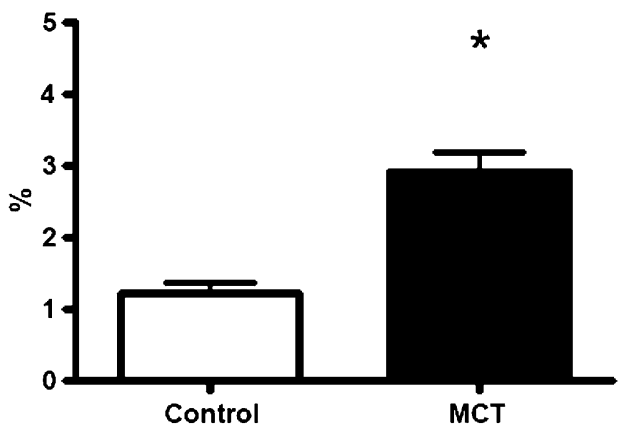

b

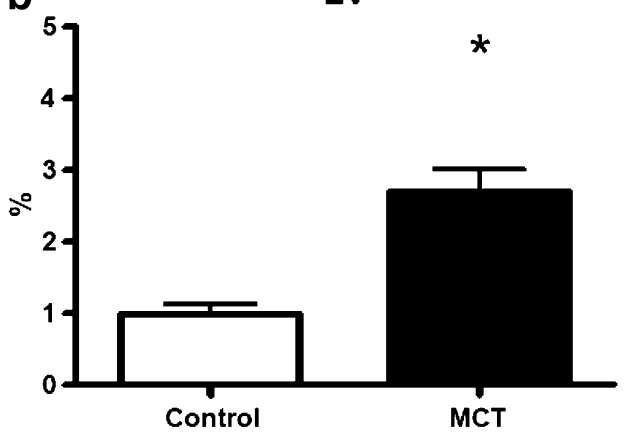

C

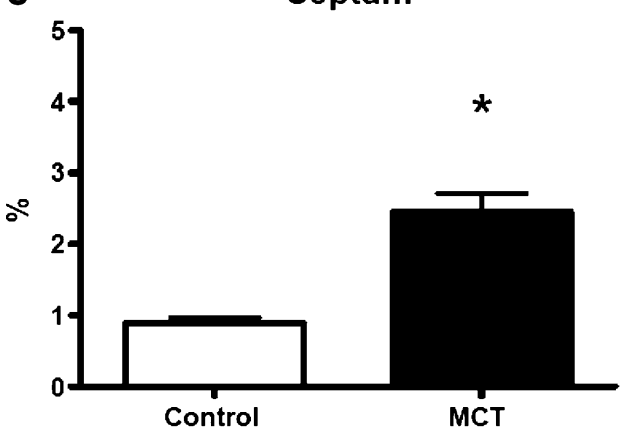

ImageJ macro

d RV

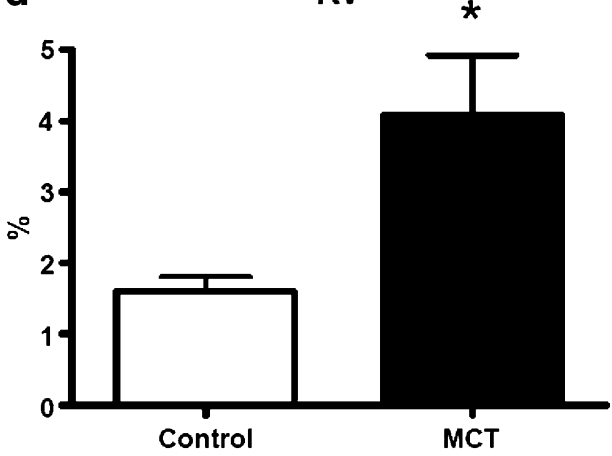

e

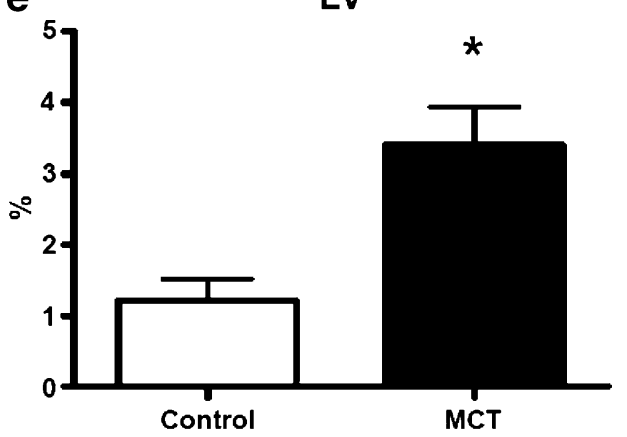

f

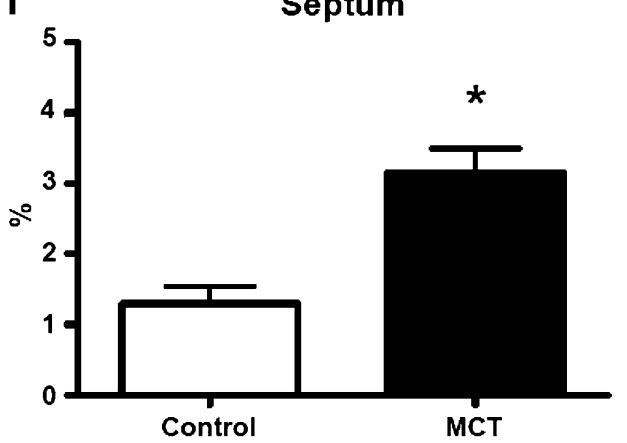


using stereology. Quantification of fibrosis using polarisation showed a significant correlation with stereology $\left(r^{2}=0.93, p<0.0001\right.$, intercept at $X=0$ is -0.09 . Fig. 5a). Quantification of fibrosis on the Mirax scanned images with ImageJ (Mirax-ImageJ) also revealed a strong correlation $\left(r^{2}=0.95, p<0.0001\right.$, intercept at $X=0$ is 0.32 . Fig. 5b). In addition, Mirax-ImageJ correlated with polarisation analysis $\left(\mathrm{r}^{2}=0.92, p<0.0001\right.$, intercept at $X=0$ is 0.37. Fig. 5c).

\subsection{Automated analyses on paraffin embedded human tissue}

We tested whether the staining technique used on frozen tissues is also applicable to paraffin embedded sections of human tissue as well as to other tissue than heart. We therefore stained sections of human myocardial tissue, lungs, kidney and liver with Picrosirius red, and scanned these with the Mirax slide scanner. Qualitative image analysis showed consistent staining of fibrosis as in myocardial tissues of rat (Fig. 6a-d). Subsequent quantitative analysis with the ImageJ macro detected fibrosis in paraffin embedded sections of human tissue of heart, lungs, kidney and liver similar to cardiac tissue of rat and a similar correlation was found with stereology $\left(r^{2}=0.91\right)$, (Fig. 7).

\subsection{Time efficiency}

Analyzing 21 areas (7 areas each of RV, LV and Septum) in sections using traditional stereology takes approximately $45 \mathrm{~min}$. It takes approximately $30 \mathrm{~min}$ to analyze the same areas using polarisation microscopy. MiraxImageJ was able to quantify fibrosis in the same areas in 10 min, including scanning and selection of regions of interest. Ergo, Mirax-ImageJ analyses was able to quantify fibrosis in ca. $22 \%$ of the time needed for stereology and $33 \%$ of the time required for polarisation microscopy.

\section{Discussion}

Introduced as prototypes only a few years ago, high resolution whole slide imaging (WSI) systems have become indispensable in the field of diagnostics and research alongside traditional microscopy. Despite their increasing potential, however, detailed literature comparing quantification technique using/between WSI and traditional microscope are scarce.

Our data shows that semi-automated quantification of fibrosis in histological samples obtained from WSI is feasible and strongly correlates with polarisation light microscopy and stereology. Although this method does not completely eliminates investigator's influence, it reduces it to a minimum since determination of the optimal threshold was done only once by analyzing a test set of 63 images. In addition, our method is time-efficient, especially when large numbers of sections have to be analyzed, and it reduces investigator dependency to a minimum. The only investigator dependent aspect is the initial selection and acquiring of images in the regions of interest (RV, LV and septum), which applies to all methods studied.

Servais et al. [38] in an earlier study reported the results of automated quantification of interstitial fibrosis in a trichrome stained sections of renal biopsies using color segmentation image analysis. However, detailed description of automated method of quantification and analysis were lacking making it hard to reproduce these results.

With respect to comparing standard methods to a new automated method, to our knowledge, only the study of Vasiljevic and co-workers, directly compared different histological methodologies used for fibrosis quantification [42]. This study, on human endomyocardial biopsies,
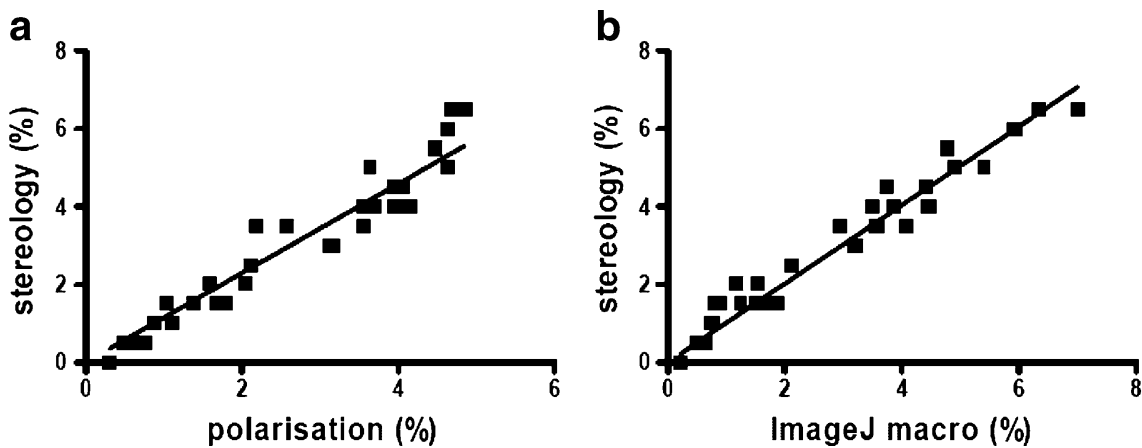

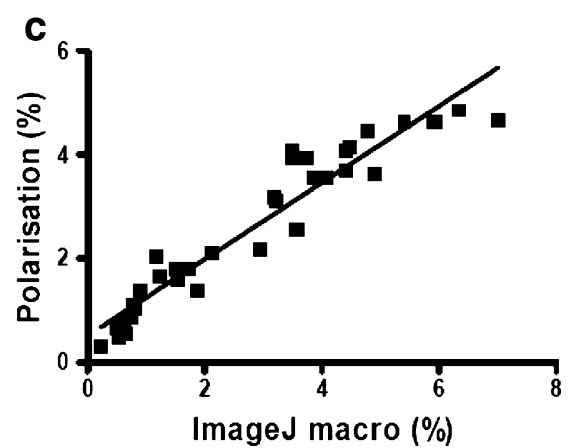

Fig. 5 We compared both analysis methods with traditional quantification of fibrosis using stereology. Both polarisation light microscopy (a) and the ImageJ macro (b) correlated strongly with stereology $\left(r^{2}=0.93, p<0.0001\right.$, intercept at $X=0$ is -0.09 and $r^{2}=$
$0.95, p<0.0001$, intercept at $X=0$ is 0.32 , respectively). In addition, the ImageJ macro (c) correlated with polarisation microscopy as well $\left(r^{2}=0.92, p<0.0001\right.$, intercept at $X=0$ is 0.37$)$ 
Fig. 6 Picrosirius red staining was also performed on human (a) myocardial tissue, (b) lung, (c) kidney and (d) liver and scanned with Mirax scan
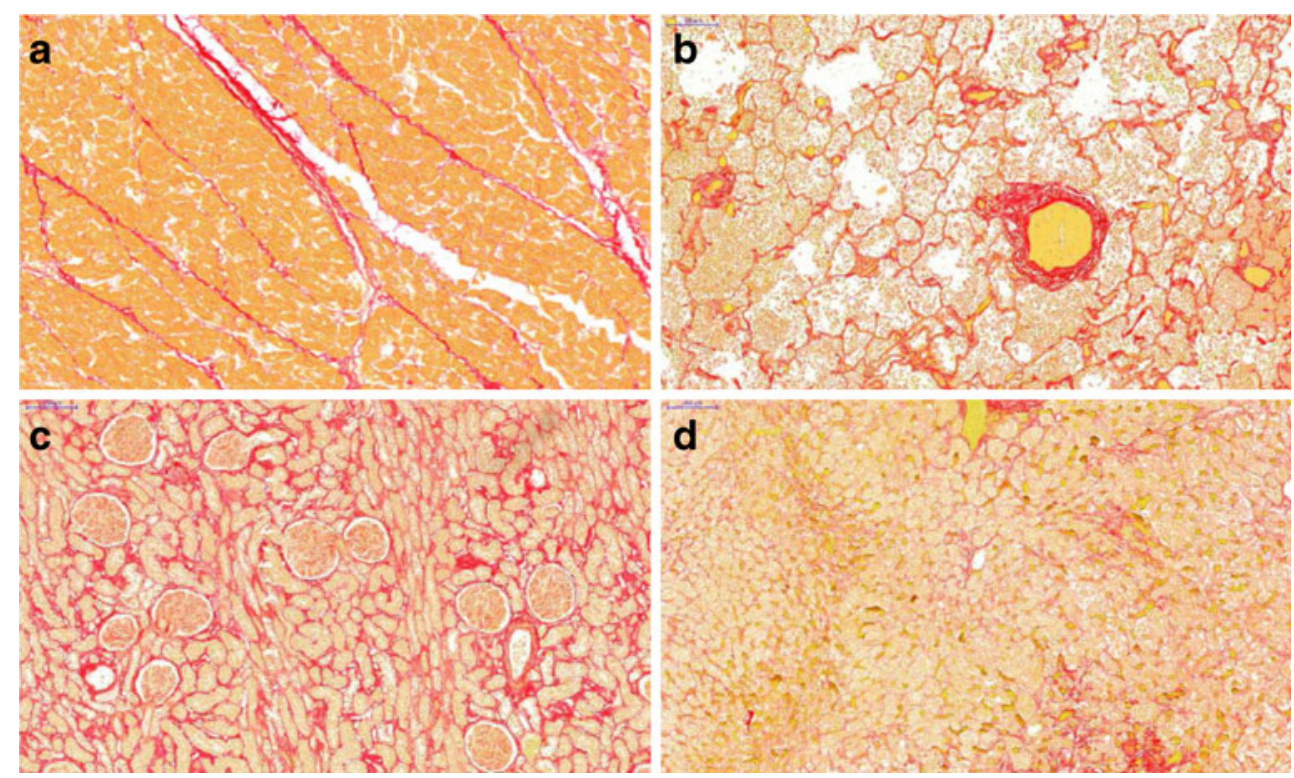

compares semi-quantitative scoring of fibrosis with computer assisted analysis and point-lesion counting using a grid. Vasiljevic et al. show correlations between different methods, however it must be noted these are to some degree subjective. In computer assisted image analysis, every section needs a threshold to identify fibrosis. In semiquantitative scoring, the investigator itself has to determine the degree of fibrosis, again in every section. In our study, stereology correlated significantly with computer assisted quantification of fibrosis, which is in accordance with Vasiljevic et al. The major difference between their method and the method presented here is that our macro automatically discriminates between fibrosis and all non-fibrotic compartments, like lumen and cardiomyoctes. Moreover, artefacts can be excluded, although this requires manual annotations in the original images. Vasiljevic also did not apply polarisation microscopy, which according to literature, is still the method of choice.

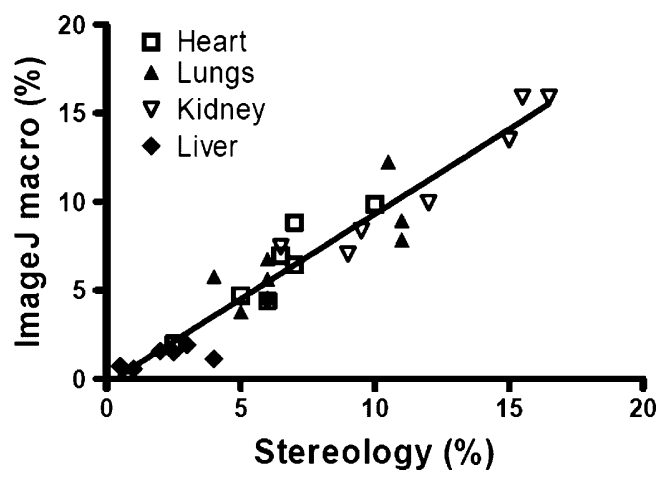

Fig. 7 The Image $J$ macro was able to detect fibrosis in paraffin embedded sections of human tissue of heart, lung, kidney and liver in the same way as in cardiac tissue of rat and a similar correlation was found with stereology $\left(r^{2}=0.91\right)$
Recently, Gaspard et al. published a detailed description of fibrosis quantification using colour subtractive image analysis in histological samples of myocardium [14]. They described how thresholding of colours in digital images can be used to discriminate between fibrosis and surrounding tissue in a mouse model of isoproteronol induced myocardial hypertrophy. In this technique, the investigator has to set thresholds for every image that is analysed, which makes it sensitive for inter-observer variation and it is laborious. Compared to the study performed by Gaspard and co-workers, our method does not need thresholding in every image the investigator wants to analyze, since the threshold are set empirically once on a test set of images. However one may need a new set of test images for each scanner used or for each staining batch. In addition, their method was not validated by comparison with other methods used for histological fibrosis quantification.

Investigators using traditional stains like van Gieson and trichrome for detecting collagen are usually confronted with the typical problems associated with automated image analyses such as variations in staining intensity, hue, contrast and the stain's tendency to fade. The fact underlying these problems is lack of precise selectivity for collagen fibers $[22,34,45]$ by these stains. In addition, qualitative studies have shown that collagen fiber structure changes with time as a function of age or as wound healing progresses $[8,30,44]$. As expected a subsequent time associated shift in color spectrum (ranging from green to orange) would be found [22]. In tissues where color change is expected to be present, separate hue (color) analyses may be necessary to study the structural details of collagen fibers.

To resolve these confounding issues, we chose Picrosirius red to stain collagen. Picrosirius red F3BA in saturated picric 
acid solution specifically and consistently stained thin collagen fibers, did not fade and was suitable for semi-automated quantification [32, 34]. In addition we took further step in our staining technique by initially incubating frozen sections of myocardial tissues in xyline for $10 \mathrm{~min}$ as described earlier. This background eliminating process proves crucial in reducing staining variations to a minimum whilst generating a clear contrast between red stained collagen fibers in a bright yellow background of cardiomyocytes.

In our study, the ImageJ macro detected fibrosis in paraffin embedded tissue and on other tissues than frozen rat heart tissue as well. However, it must be noted that these tissues were examined with thresholds set for cardiac rat tissue. In principle the macro can be reused on any kind of histological staining in any kind of tissue, as long as structures in the macro are defined properly and thresholds are set accordingly.

Finally, it is worth noting that the ImageJ macro analysis detected more fibrosis than the polarization filter method (Figs. 4 and 5). This outcome is consistent with the study conducted by Diaz Encarnacion and colleagues [11] in which collagen content was assessed and compared using 3 methods; Mason's trichome, Picrosirius red with and without linearly polarized light. The highest collagen content was obtained in Picrosirius red stained sections without polarized light.

We scanned the sections with a Mirax scanner enabling us to digitally store histological sections for infinite period of time and for easy future references, allowing simultaneous viewing of whole slide histopathology and selection of regions for measurement. Essentially any image capturing device such as CCD camera could be employed for the acquisition of digital images of sections. Subsequent analytical measurements with our in house developed macro can be performed in an automated fashion by ImageJ. The macro can also be rewritten for commercially available software like ImagePro or Matrox Inspector.

We chose to investigate fibrosis in a rat model of $\mathrm{PH}$, induced by monocrotaline, which is one of the most commonly used models to investigate RV pressure overload, and found a significant increase of collagen content in the RV of PH rats compared to controls. In our study however, fibrosis was not limited to the RV as it was also observed in the LV of these hearts. Lourenco et al. in an earlier study of monocrotaline induced rat model of $\mathrm{PH}$ also reported a similar observation [24]. In addition, they also observed that RV fibrosis was significantly higher compared to LV. In our study however, we could not demonstrate such significant difference. Whereas Lourenco and co-workers sacrificed experimental animals after 6 weeks, ours were sacrificed after 3 weeks. Interestingly, in their previous work [19] Lourenco and co-workers using the same experimental model reported an already estab- lished RV hypertrophy after 3 weeks. It is known in experimental models that right ventricular hypertrophy is accompanied by increased formation of myocardial fibrosis $[20,26]$. Moreover, as observed earlier, collagen fibers are expected to change as function of age or as wound healing progresses. It is also known that monocrotaline induced $\mathrm{PH}$ activates neurohumoral factors, which can also influence structural remodeling of the $\mathrm{LV}$, in the absence of mechanical overload $[1,23,27]$. In addition, vice versa, the RV also shows severe fibrosis in the setting of systemic hypertension [4, 39, 47].

\section{Conclusion}

We have demonstrated that the combination of unique Picrosirius red staining technique and a well defined macro based algorithm for semi-automated WSI analysis provides a powerful tool for rapid quantification of myocardial fibrosis. This will provide researchers with useful information for monitoring the severity of myocardial fibrosis and identifying rationale targets for interventions.

The proposed method to quantify collagen correlates excellently with the traditional methods is time-efficient and may prove less user dependent as far as thresholding is concerned.

Acknowledgements This work was supported by: Institute for Cardiovascular Research, VU University Medical Center, Amsterdam

Open Access This article is distributed under the terms of the Creative Commons Attribution Noncommercial License which permits any noncommercial use, distribution, and reproduction in any medium, provided the original author(s) and source are credited.

\section{References}

1. F. Akhavein, E.J. St-Michel, E. Seifert, C.V. Rohlicek, Decreased left ventricular function, myocarditis, and coronary arteriolar medial thickening following monocrotaline administration in adult rats. J Appl Physiol 103, 287-295 (2007)

2. B.C. Berk, K. Fujiwara, S. Lehoux, ECM remodeling in hypertensive heart disease. J Clin Invest 117, 568-575 (2007)

3. A. Borbely, V.Z. d van Papp, J.G. Bronzwaer, I. Edes, G.J. Stienen, W.J. Paulus, Cardiomyocyte stiffness in diastolic heart failure. Circulation 111, 774-781 (2005)

4. C.G. Brilla, R. Pick, L.B. Tan, J.S. Janicki, K.T. Weber, Remodeling of the rat right and left ventricles in experimental hypertension. Circ Res 67, 1355-1364 (1990)

5. B. Burstein, S. Nattel, Atrial fibrosis: mechanisms and clinical relevance in atrial fibrillation. J Am Coll Cardiol 51, 802-809 (2008)

6. D. Chemla, V. Castelain, M. Humbert, J.L. Hebert, G. Simonneau, Y. Lecarpentier, P. Herve, New formula for predicting mean pulmonary artery pressure using systolic pulmonary artery pressure. Chest 126, 1313-1317 (2004) 
7. C.F. Chesney, J.R. Allen, Animal model: pulmonary hypertension, cor pulmonale and endocardial fibroelastosis in monocrotalineintoxicated nonhuman primates. Am J Pathol 70, 489-492 (1973)

8. C.R.G. Debessa, L.B.M. Maifrino, R.R. de Souza, Age related changes of the collagen network of the human heart. Mech Ageing Dev 122, 1049-1058 (2001)

9. A.L. des Tombe, B.J. van Beek-Harmsen, M.B. Lee-de Groot and W.J. van der Laarse, Calibrated histochemistry applied to oxygen supply and demand in hypertrophied rat myocardium, Microsc. Res.Tech. 58 (2002), 412-420.

10. G. Desimone, D.C. Wallerson, M. Volpe, R.B. Devereux, Echocardiographic Measurement of Left-Ventricular Mass and Volume in Normotensive and Hypertensive Rats-Necropsy Validation. Am J Hypertens 3, 688-696 (1990)

11. M.M. Diaz Encarnacion, M.D. Griffin, J.M. Slezak, E.J. Bergstralh, M.D. Stegall, J.A. Velosa, J.P. Grande, Correlation of quantitative digital image analysis with the glomerular filtration rate in chronic allograft nephropathy. Am J Transplant 4, 248-256 (2004)

12. T.H. Everett, J.E. Olgin, Atrial fibrosis and the mechanisms of atrial fibrillation. Heart Rhythm 4, S24-S27 (2007)

13. M.W. Frohlich, Birefringent objects visualized by circular polarization microscopy. Stain Technol 61, 139-143 (1986)

14. G.J. Gaspard, K.B. Pasumarthi, Quantification of cardiac fibrosis by colour-subtractive computer-assisted image analysis. Clin Exp Pharmacol Physiol 35, 679-686 (2008)

15. M. Gautier, D. Antier, P. Bonnet, J.L. Le Net, G. Hanton, V. Eder, Continuous inhalation of carbon monoxide induces right ventricle ischemia and dysfunction in rats with hypoxic pulmonary hypertension. Am J Physiol Heart Circ Physiol 293, H1046H1052 (2007)

16. M.L. Handoko, I. Schalij, K. Kramer, A. Sebkhi, P.E. Postmus, W. J. van der Laarse, W.J. Paulus, A. Vonk-Noordegraaf, A refined radio-telemetry technique to monitor right ventricle or pulmonary artery pressures in rats: a useful tool in pulmonary hypertension research. Pflugers Arch 455, 951-959 (2008)

17. M. Hardziyenka, M.E. Campian, H.A. de Bruin-Bon, M.C. Michel, H.L. Tan, Sequence of echocardiographic changes during development of right ventricular failure in rat. J Am Soc Echocardiogr 19, 1272-1279 (2006)

18. I.R. Henkens, K.T. Mouchaers, H.W. Vliegen, W.J. van der Laarse, C.A. Swenne, A.C. Maan, H.H. Draisma, I. Schalij, E.E. van der Wall, M.J. Schalij, A. Vonk-Noordegraaf, Early changes in rat hearts with developing pulmonary arterial hypertension can be detected with three-dimensional electrocardiography. Am J Physiol Heart Circ Physiol 293, H1300-H1307 (2007)

19. T. Henriques-Coelho, J. Correia-Pinto, R. Roncon-Albuquerque Jr., M.J. Baptista, A.P. Lourenco, S.M. Oliveira, A. BrandaoNogueira, A. Teles, J.M. Fortunato, A.F. Leite-Moreira, Endogenous production of ghrelin and beneficial effects of its exogenous administration in monocrotaline-induced pulmonary hypertension. Am J Physiol Heart Circ Physiol 287, H2885-H2890 (2004)

20. M. Honda, S. Yamada, Y. Goto, S. Ishikawa, H. Yoshikane, Y. Ishinaga, H. Kuzuo, S. Morioka, K. Moriyama, Biochemical and structural remodeling of collagen in the right ventricular hypertrophy induced by monocrotaline. Jpn Circ J 56, 392-403 (1992)

21. L.C. Junqueira, G. Bignolas, R.R. Brentani, Picrosirius staining plus polarization microscopy, a specific method for collagen detection in tissue sections. Histochem J 11, 447-455 (1979)

22. J.A. Kiernan Methods for connective tissue. in: J. A. Kiernan (Ed.), Histological and Histochemical Methods, Arnold, London, 2002, pp. 144-163.

23. R.R. Lamberts, R.J. Vaessen, N. Westerhof, G.J. Stienen, Right ventricular hypertrophy causes impairment of left ventricular diastolic function in the rat. Basic Res Cardiol 102, 19-27 (2007)
24. A.P. Lourenco, R. Roncon-Albuquerque Jr., C. Bras-Silva, B. Faria, J. Wieland, T. Henriques-Coelho, J. Correia-Pinto, A.F. Leite-Moreira, Myocardial dysfunction and neurohumoral activation without remodeling in left ventricle of monocrotaline-induced pulmonary hypertensive rats. Am J Physiol Heart Circ Physiol 291, H1587-H1594 (2006)

25. S. Mito, R. Ozono, T. Oshima, Y. Yano, Y. Watari, Y. Yamamoto, A. Brydun, K. Igarashi, M. Yoshizumi, Myocardial protection against pressure overload in mice lacking Bach1, a transcriptional repressor of heme oxygenase-1. Hypertension 51, 1570-1577 (2008)

26. S. Morioka, M. Honda, S. Ishikawa, Y. Ishinaga, S. Yano, K. Tanaka, K. Moriyama, Changes in contractile and non-contractile proteins, intracellular $\mathrm{Ca} 2+$ and ultrastructures during the development of right ventricular hypertrophy and failure in rats. Jpn Circ J 56, 469-474 (1992)

27. K.T. Mouchaers, I. Schalij, A.M. Versteilen, A.M. Hadi, G.P. van Nieuw Amerongen, V.W. van Hinsbergh, P.E. Postmus, W.J. van der Laarse, A. Vonk-Noordegraaf, Endothelin receptor blockade combined with phosphodiesterase-5 inhibition increases right ventricular mitochondrial capacity in pulmonary arterial hypertension. Am J Physiol Heart Circ Physiol 297, H200-H207 (2009)

28. A. Nicoletti, D. Heudes, C. Mandet, N. Hinglais, J. Bariety, J.B. Michel, Inflammatory cells and myocardial fibrosis: spatial and temporal distribution in renovascular hypertensive rats. Cardiovasc Res 32, 1096-1107 (1996)

29. F. Pelliccia, G. d'Amati, C. Cianfrocca, P. Bernucci, A. Nigri, B. Marino, P. Gallo, Histomorphometric features predict 1-year outcome of patients with idiopathic dilated cardiomyopathy considered to be at low priority for cardiac transplantation. Am Heart J 128, 316-325 (1994)

30. J.G. Pickering, C.M. Ford, L.H. Chow, Evidence for rapid accumulation and persistently disordered architecture of fibrillar collagen in human coronary restenosis lesions. Am J Cardiol 78, 633-637 (1996)

31. J.F. Pombo, B.L. Troy, R.O. Russell Jr., Left ventricular volumes and ejection fraction by echocardiography. Circulation $\mathbf{4 3}, 480$ 490 (1971)

32. H. Puchtler, F.S. Waldrop, L.S. Valentine, Polarization microscopic studies of connective tissue stained with picro-sirius red FBA. Beitr Pathol 150, 174-187 (1973)

33. M.A. Quinones, C.M. Otto, M. Stoddard, A. Waggoner, W.A. Zoghbi, Recommendations for quantification of Doppler echocardiography: a report from the Doppler Quantification Task Force of the Nomenclature and Standards Committee of the American Society of Echocardiography. J Am Soc Echocardiogr 15, 167-184 (2002)

34. L. Rich., Whittaker P., Collagen and Picrosirius Red staining: a polarized light assessment of fibrillar hue and spatial distribution, Braz.J.Morphol.Sci. 22 (2005), 97-104.

35. V.M. Salemi, A.M. Bilate, F.J. Ramires, M.H. Picard, D.M. Gregio, J. Kalil, E.C. Neto and C. Mady, Reference values from M-mode and Doppler echocardiography for normal Syrian hamsters, Eur.J Echocardiogr. 6 (2005), 41-46.

36. R.T. Schermuly, K.P. Kreisselmeier, H.A. Ghofrani, H. Yilmaz, G. Butrous, L. Ermert, M. Ermert, N. Weissmann, F. Rose, A. Guenther, D. Walmrath, W. Seeger, F. Grimminger, Chronic sildenafil treatment inhibits monocrotaline-induced pulmonary hypertension in rats. Am J Respir Crit Care Med 169, 39-45 (2004)

37. U. Seeland, S. Selejan, S. Engelhardt, P. Muller, M.J. Lohse, M. Bohm, Interstitial remodeling in beta1-adrenergic receptor transgenic mice. Basic Res Cardiol 102, 183-193 (2007)

38. A. Servais, V. Meas-Yedid, M. Buchler, E. Morelon, J.C. OlivoMarin, E. Thervet, Quantification of interstitial fibrosis by image analysis on routine renal biopsy 1 year after transplantation in patients managed by $\mathrm{C} 2$ monitoring of cyclosporine microemulsion. Transplant Proc 39, 2560-2562 (2007) 
39. F.G. Spinale, Myocardial matrix remodeling and the matrix metalloproteinases: influence on cardiac form and function. Physiol Rev 87, 1285-1342 (2007)

40. L. van Heerebeek, N. Hamdani, M.L. Handoko, I. Falcao-Pires, R.J. Musters, K. Kupreishvili, A.J. Ijsselmuiden, C.G. Schalkwijk, J.G. Bronzwaer, M. Diamant, A. Borbely, d. van, V, G.J. Stienen, G.J. Laarman, H.W. Niessen, W.J. Paulus, Diastolic stiffness of the failing diabetic heart: importance of fibrosis, advanced glycation end products, and myocyte resting tension, Circulation 117, 43-51 (2008)

41. K.H. van Hoeven, S.M. Factor, A comparison of the pathological spectrum of hypertensive, diabetic, and hypertensive-diabetic heart disease. Circulation 82, 848-855 (1990)

42. J.D. Vasiljevic, Z.B. Popovic, P. Otasevic, Z.V. Popovic, R. Vidakovic, M. Miric, A.N. Neskovic, Myocardial fibrosis assessment by semiquantitative, point-counting and computer-based methods in patients with heart muscle disease: a comparative study. Histopathology 38, 338-343 (2001)
43. K.T. Weber, Fibrosis and hypertensive heart disease. Curr Opin Cardiol 15, 264-272 (2000)

44. P. Whittaker, D.R. Boughner, R.A. Kloner, Analysis of healing after myocardial infarction using polarized light microscopy. Am J Pathol 134, 879-893 (1989)

45. P. Whittaker, R.A. Kloner, D.R. Boughner, J.G. Pickering, Quantitative assessment of myocardial collagen with picrosirius red staining and circularly polarized light. Basic Res Cardiol 89, 397-410 (1994)

46. T.A. Wynn, Cellular and molecular mechanisms of fibrosis. J Pathol 214, 199-210 (2008)

47. M. Young, M. Fullerton, R. Dilley, J. Funder, Mineralocorticoids, hypertension, and cardiac fibrosis. J Clin Invest 93, 2578-2583 (1994)

48. W. Zhao, T. Zhao, Y. Chen, R.A. Ahokas, Y. Sun, Oxidative stress mediates cardiac fibrosis by enhancing transforming growth factor-beta1 in hypertensive rats. Mol Cell Biochem 317, 43-50 (2008) 